\title{
IP-based RFID Location System
}

\author{
Phuoc Nguyen Tran and Nadia Boukhatem \\ Computer Science and Network Department, Telecom ParisTech \\ 46 rue Barrault, 75013 Paris, \\ France
}

\section{Introduction}

The Radio Frequency Identification (RFID) landscape has been radically changing since decades. It has been widely deployed by commercial and industrial organizations as well as government agencies with a wide range of applications. The RFID technology makes it possible to identify an object, to track it and learn its characteristics remotely, thanks to a label emitting radio waves, integrated or attached to the object. The RFID technology enables reading of labels even without direct line of sight and can pass through thin layers of material (paint, snow, etc.).

In the last few years, the RFID systems have evolved significantly in terms of technology and cost, enabling the RFID systems to stand out as the reference identification technology in numerous fields of applications such as asset tracking, logistics and supply chain management, animal tracking, healthcare, warehouse management, manufacturing engineering, automotive, contactless payments, etc. and mandated by industry giants (e.g. Wall-Mart, Target, Tesco and Albertson, etc.) and various government agencies (e.g. U.S. Department of Defense and Department of Homeland security, etc.).

One of the main advantages of the RFID technology is to provide a low cost and easy to install indoor location system compared to other positioning systems, such as Global Positioning System (GPS), Wi-Fi, Ultra-Wideband (UWB), Infrared (IR), and sensor based systems, etc.

A number of location sensing systems based on RFID technology have been proposed for indoor location services. SpotON [high] supports indoor location service using RFID technology based on radio signal strength analysis. LANMARC [ni] aims at increasing the accuracy in determining the RFID tag location and economizing the deployment cost of the system. To increase the accuracy, the system defines extra fixed location reference tags to help location calibration. In addition, an algorithm which reflects the relations of signal strengths by power level is developed in order to compute accurately the physical distance between the objects and the reader. FLEXOR [sue] is an improvement of LANDMARC to reduce the computational overhead in determining the location of the objects. The system divides the location area into cells which reduce the information used for localization calculation and decreases the computation load for the localization service. FERRET [liu] allows not only locating the RFID tagged objects, but also displaying the objects. The system uses an RFID reader embedded in a camera. FERRET uses also the algorithm which reflects the relations of signal strengths by power level to locate the objects. When the object is

Source: Radio Frequency Identification Fundamentals and Applications, Bringing Research to Practice, Book edited by: Cristina Turcu, ISBN 978-953-7619-73-2, pp. 278, February 2010, INTECH, Croatia, downloaded from SCIYO.COM 
found, the camera is turned on and displays their locations in real-time. In [teso], a sensing surface location system is proposed. The system is capable of tracking the objects within a closed environment. The objects (e.g devices, people, robots, ect.) integrate passive RFID readers. The RFID tags are installed as a grid representing the sensing surface. When the objects are on the surface, their locations are determined by mapping the RFID tag detected by the RFID readers and the physical surface of the RFID tags that it represents.

Besides, some solutions use Wi-Fi based Active RFID tags to expand the coverage zone using the Wi-Fi technology and provide complete wireless asset tracking and monitoring [aero] [ekah].

Considering the RFID management systems, the EPC global network developed by EPCglobal [jud][klau][epc] is the most representative. EPCglobal aims at standardizing the electronic product code (EPC) and the automatic identification system in the specific context of supply chain.

With the target to develop open RFID management systems and ease the integration of existing network services along with RFID location functionality, a number of works have been proposed.

SOA-oriented networked RFID system [zhang] proposes RFID location tracking service using web services. This system enables a distributed deployment of RFID solution which hides the diversity of the RFID hardware and underlying I/O communication protocols. Other works have investigated the use of session initiation protocol (SIP) [sip] as a control protocol for RFID management. SIP-based RFID management system (SRMS) [cho] uses SIP to manage the RFID tags. SRMS enhances the existing SIP architecture to perform RFID tags location registration and tracking procedures.

Our work is motivated by developing an IP-based RFID system management thus enabling low cost and large scale deployment of the RFID technology as well as openness and ease of integration with the existing IP-based services. In particular, a SIP implementation will have the benefit of providing a flexible integration of RFID location with the other corporate network services

Several motivations underpin our work:

- $\quad$ Propose a SIP-based RFID location system for indoor location. The current location of an object is determined by the identity of the reader when the object moves under its coverage zone.

- Integrate the RFID location services to the other IP services of the corporate network. This provides an integrated and flexible solution.

- More and more communication services such as location based services (LBS) are developed using the location information. The RFID based location system constitutes a good basis for providing such customized services.

\subsection{Positioning systems}

A positioning system allows determining the location of an object in space based on realtime collected information. It is also able to track the current location of an object moving through a space.

GPS system [joy][ivan] is today a widely deployed system. It provides the location of an object on the Earth (e.g. latitude and longitude). The current location of the objects moving through space could be visual using navigating map provided by the GPS providers. The GPS system is consisting of satellites in located geostationary orbit around the Earth and the GPS devices sending the updated location information to the satellites. If GPS seems to be a 
good solution for the development of outdoor location systems, it is not able to be deployed inside buildings which impair the signal reception. It performs only in area where path between the GPS receiver and the sky is unobstructed. Moreover, the GPS devices require a dedicated chipset, a ground GPS system to extend signal range in large areas leading to a very costly deployment.

With the widespread adoption of wireless LANs, the Wi-Fi technology has been adopted as a tool for developing indoor positioning systems [joy][aero][ekah]. The Wi-Fi positioning system reuses the existing Wi-Fi infrastructures including the Wi-Fi access points, Wi-Fibased radio tags integrated in the mobile devices and specific software allowing the system administrators to track the device location and analyze the position accuracy. The location of the devices is detected by measuring the signal strength indicator (RSSI). Although reusing the existing Wi-Fi infrastructure for developing the positioning system economizes the deployment cost, the Wi-Fi network cards and the management system deployment constitutes a non negligible cost.

UWB-based positioning system determines the current location of the object by scanning and continuously monitoring the UWB radio transceivers attached to clients [joy] [edoc]. The UWB-based system uses radio signal in a very wide bandwidth. The position calculation is based on time-of-arrival (TOA) technique. The main advantage of the UWB system is that it can be used at very low energy levels for short-range high-bandwidth communications. The issue is that the battery replacement constitutes a significant cost.

An object can be detected by an IR-based system [edoc] when it is present in the signal range of the IR detectors. The IR-based system enables to make a real-time tracking but it suffers from some shortcomings. First, it is not able to detect the object without direct line of sigh. The IR signal degrades when there is material obstacle such as walls, ceilings, and carton boxes because the signal cannot penetrate opaque materials. The IR tags and the IR detectors have to be installed everywhere to avoid loosing tracked objects. In addition, the IR system suffers from its sensitiveness to sunlight, direct light of sight requirement, cost of installation and maintenance at large scale.

Similar to most other positioning solutions, sensor-based systems [edoc] allow determining the location of the objects (tags) by using the Time Difference of Arrival (TDOA) technique. Each object has to integrate a battery-powered radio module that transmit frequently RF signal of its unique identification number. The sensors are installed to receive the RF signal. The object location is calculated by TDOA using the triangulation method based on the analysis of radio signal strength. However, the triangulation method using TDOA cannot provide an accurate position when the signal from the sensors to the tag is obstructed. Therefore, many sensor based systems are not widely deployed. Moreover, the obligation of battery integration into the tags limits the sensor network deployment capacities in terms of easy installation and cost.

The RFID technology allows locating the RFID tags attached to object at a close distance that use radio frequency (RF). These tags emit messages readable by RFID readers. Each RFID tag contains a unique identification number. There are two general categories of the RFID tags, active and passive, depending on their source of electrical power. Active RFID tags contain usually their own power source. Passive tags are powered from the signal of the readers. The RFID readers are also categorized into two general types: active and passive. Each type of reader can read specific type of tag [wein].

Compared to other positioning systems, the RFID technology provides following advantages: 
- Covers indoor areas - fewer limitations compared to outdoor positing system such as GPS. In addition, the RFID technology can provide indoor positioning without any considerations of the direct line of sight unlike the other indoor positioning systems such as IR, Wi-Fi and sensor based systems.

- Quick installation -the investments can be quickly returned by installing the RFID system in days or weeks rather than months or years.

- Cost effective - indoor location systems such as Wi-Fi, UBW, and sensor based require battery installation. The RFID technology through using passive tags economizes the deployment cost.

\subsection{RFID management systems}

EPCglobal is an industry-driven organization which aims at standardizing the electronic product code (EPC) and the automatic identification system in the supply chain. The EPC code stored in the RFID tag allows uniquely identifying a physical object. EPCglobal defines EPCIS (EPC information system) for object identification. The EPCIS includes several components that allow accessing/exchanging information between the enterprises subscribing to the EPCglobal network. However, EPCglobal network is designed as a middleware solution with a specific goal.

While the Internet today is recognized as a network that is fundamentally changing social, political, and economic structures, the trend is that all network technologies converge to IP (Internet Protocol).

The term "Internet of Things" is a new notion that describes a number of technologies and research disciplines that enable the Internet to reach out into the real world of physical objects [itu]. The RFID technology is at the forefront of the Internet of Things. Today, it is one of the most promising automated identification technologies; by sticking an RFID tag to a physical object, it can be identified as a unique entity in the virtual world making the internet of things a reality.

Location awareness is a key feature of the ubiquitous computing, one of the advanced concepts of the Internet of things.

Many initiatives have been launched to develop open RFID based systems supporting location tracking.

The SOA-oriented networked RFID system [zhang] proposes a decentralized and plate-form independent location-tracking services using web services technology. The system provides a modular and layered application framework allowing scalability and extensibility. SIP-based RFID management system (SRMS) [cho] uses session initiation protocol (SIP) [sip], which is an Internet standard protocol for session initiation management to manage the RFID tags. SRMS enhances the existing SIP architecture by introducing a surrogate user agent (SUA) and a SRMS name server (SNS). The SUA performs location registration procedures on behalf of RFID tags with limited capabilities, while the SNS provides name resolution services for location registration and tracking of RFID tags. The RFID-enabled location tracking system (SIP-RLTS) [zang] also based on SIP is proposed to support the location management. The SIP-RLTS solution uses the SIP event notification model to support the PUSH and PULL operation needed by the location service.

\subsection{Contributions and organization}

The contributions of this chapter are as follows: 
- $\quad$ Design and specify an IP-based RFID architecture for location management.

- The location service implements two main functions: location and tracking. The location function aims at registering the current location of the RFID objects while the tracking function returns their current location.

- The implementation system defines an interface, which interconnects the RFID system to the existing communication infrastructure and services. With this interface, the location system is notified the RFID objects located in the detection area of the RFID reader, via entering and leaving events.

- The validation of the IP-based RFID location management system using the Session Initiation Protocol (SIP) [sip] is presented.

The chapter is organized as follows. Section 2 presents a motivating scenario that shows the benefits of location management services in daily life. In section 3, we identify different requirements and functions of the IP-based RFID architecture. The SIP-based implementation is presented in section 4 . Section 5 concludes the chapter with further works.

\section{Application scenario}

In this section, we present an application scenario to highlight the motivations of the service location development.

Consider a hospital environment where the staff has a business (or access) card that incorporates an RFID tag. An RFID tag can also be added to any medical equipment (defibrillators, portable scanner, etc.). RFID readers are scattered throughout the grounds of the hospital mainly at strategic locations or crossing such as doors. Doctors may have to move between different departments and buildings and thus among several RFID readers. The latter, through the tracking system, update their current location. If a nurse, for example, seeks to reach a doctor, she can use the location system to identify where the doctor is. The same goes for finding medical equipment more quickly.

\section{The IP-based RFID architecture}

\subsection{Architecture requirements}

As a first step of our work, we identify the requirements of the IP-based RFID location system. As presented above, the RFID technology is today considered as a cost effective method for indoor location management. However, the RFID management systems are dedicated, relatively expensive and do not inter-operate with the classical communication devices such as PDA, mobile phone, smart-phone, laptop, etc. In our context, we aim at providing a communication infrastructure which integrates both the tagged devices (RFID) and classical devices, and defines a location system based on RFID. From an operational point of view, the communication infrastructure must provide a location service based on RFID and may support the following requirements:

\subsubsection{Location function}

The location function should allow any objects to indicate their current location. The RFID readers play a significant role in detecting the tagged objects under their detection zone.

We should distinguish two types of location. Network location - which indicates the IP address of the RFID reader the tagged object is under coverage area. In our system the reader IP address of the servers also as an identity of the reader. Geographical location - 
which indicates where, geographically, the object is. The geographical location indicate the name of the room where the RFID reader is (e.g., the reader IPX is in the room 234 of the building C). A more accurate position in the form of 3D coordinates may be provided using, for example, sophisticated location mechanisms involving many readers.

Note that, it should be possible to derive from the network location the geographical location and vice versa. Thus, an RFID reader name should be associated to its IP address.

To determine the current location of an object, the location-tracking system must provide a means for recording and maintaining the current location. A database location should be installed.

Moreover, the RFID readers must support the registration procedure that allows registering the RFID tags at the location database when it enters the RFID reader coverage zone. Thus, when a RFID reader detects that an object has entered its detection zone, it must initiate a register request, in particular, indicate the corresponding <RFID tag, IP address of the RFID reader $>$. The system must also support functions to notify a particular object has left the detection zone of the reader.

\subsubsection{Tracking function}

This function allows the system to determine the current location of the object. It returns, in particular, its current location.

\subsubsection{Integration of the RFID system to existing communication infrastructure}

To seamlessly integrate the RFID system to the communication infrastructure and associated services, a communication interface between the reader and the communication infrastructure must be defined. Once a new tagged object is detected by the reader, the interface must detect this event and initiate a registration request. If a tagged object leaves the reader coverage areas, a de-registration must be initiated. Concretely, the communication interface functions should either be integrated into the reader if available resources are provided or exist independently.

\subsection{Functional architecture}

Based on the requirements above, we define a functional architecture of the RFID location system (figure 1).

The architecture includes the following:

- $\quad$ RFID Readers and RFID tags: RFID readers are installed to read information from RFID tags. The readers transmit the information obtained to RFID middleware.

- RFID middleware performs the collecting and filtering functions. The RCOM interface ensures the integration of the RFID system to the communication infrastructure. It notifies that an object enters the reader detection area or leaves it.

- Location service includes two functions: location and tracking. These functions interact with the location database which, in particular, is responsible of inferring the current location of the object after a tracking request.

\subsection{Performance and security}

Scalability is a significant feature of an RFID based system that usually runs a very large number of tags. The system must be designed so that its performance is not altered when the number of tagged items to be processed increases. 

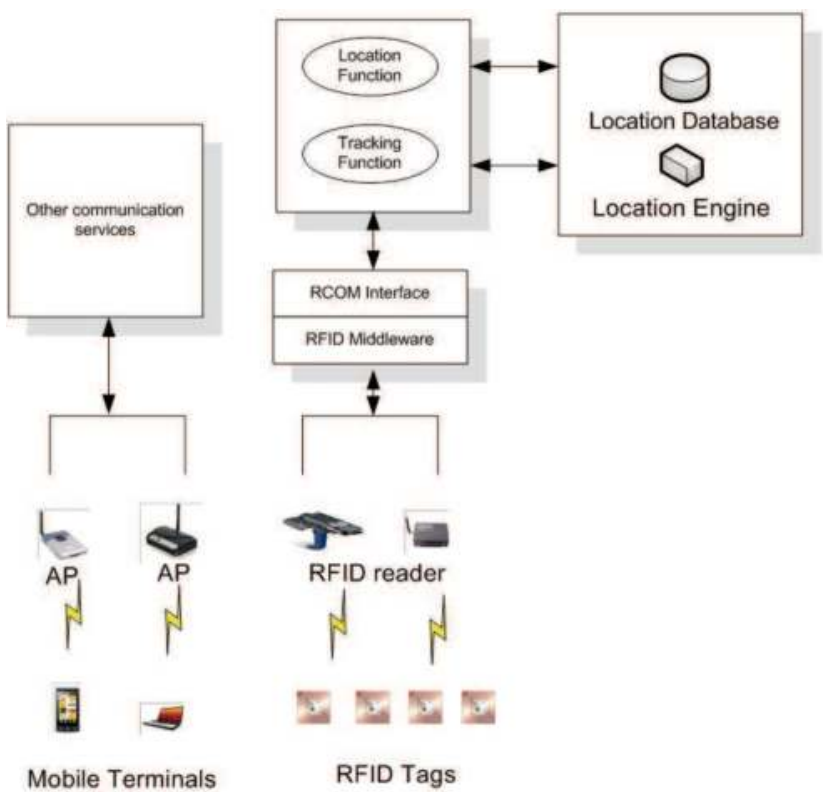

Fig. 1. Functional architecture of RFID based location system

As shown in Figure 1, the architecture of the location system is relatively centralized around the location server and the system performance is highly depended on the capacity of this server (CPU, memory, bandwidth). This capacity could be, for example, estimated by the number of queries per second that the server can process.

The location system requires various needs in terms of security. Firstly, accessing the location service should be provided only to authorized users. In addition, the location information exchange must be secured, in particular, the messages between the location server and the readers (e.g., the registration functions). The security of data transfer between the tags and readers should also be ensured. .

\section{SIP-based implementation}

In order to implement the location system specified above we choose to use the session initiation protocol (SIP) for several reasons:

- $\quad$ SIP allows easy implementation of the RFID based location service.

- $\quad$ SIP is widely used for VoIP (voice Over IP) services which are today an integral part of the corporate communication infrastructure. Assuming that a VoIP infrastructure is already deployed, our location service can easily be integrated into the infrastructure with a low cost deployment.

\subsection{SIP model overview}

The SIP architecture model is based on the concepts of SIP server (s) and SIP user agents (UA). An UA is a software entity that initiates SIP requests (UAC: UA Client) and returns SIP responses (UAS: UA Server). A SIP server can implement one or several of the following functions: registration, proxy, and redirect. 
A registration server or Registrar is a server that accepts SIP requests (e.g. SIP REGISTER) and the role of which is to register the current location of the UA.

A proxy server's role is to route SIP messages. For this purpose, the proxy server uses the SIP destination represented by an URI (Uniform Resource Identifier) and the location service to determine the current location of the destination.

A redirect server is a server that responds to a SIP INVITE request by sending a $3 x x$ message type to indicate the UA the current location of the destination to reach. Unlike proxy server, redirect server does not route SIP requests, it indicates the UA the location where it can route the request.

Note that a UA must register its current location prior to initiate a SIP session.

\subsection{SIP-based location system architecture}

The application of the SIP model to the specification described above requires the RCOM interface to behave as a SIP UA. A SIP server that performs the Registrar function has to be defined to handle the registration requests initiated by the UA. The SIP server interacts with the location database to record the current location.

To support the tracking function, the SIP server should integrate proxy and redirect functions. The redirect function is used when there is a Tracking request. The redirect server responds by indicating the current location using a $3 x x$ response type. The proxy function occurs when the tracked object is managed by other SIP servers.

The system architecture is shown in Figure 2.

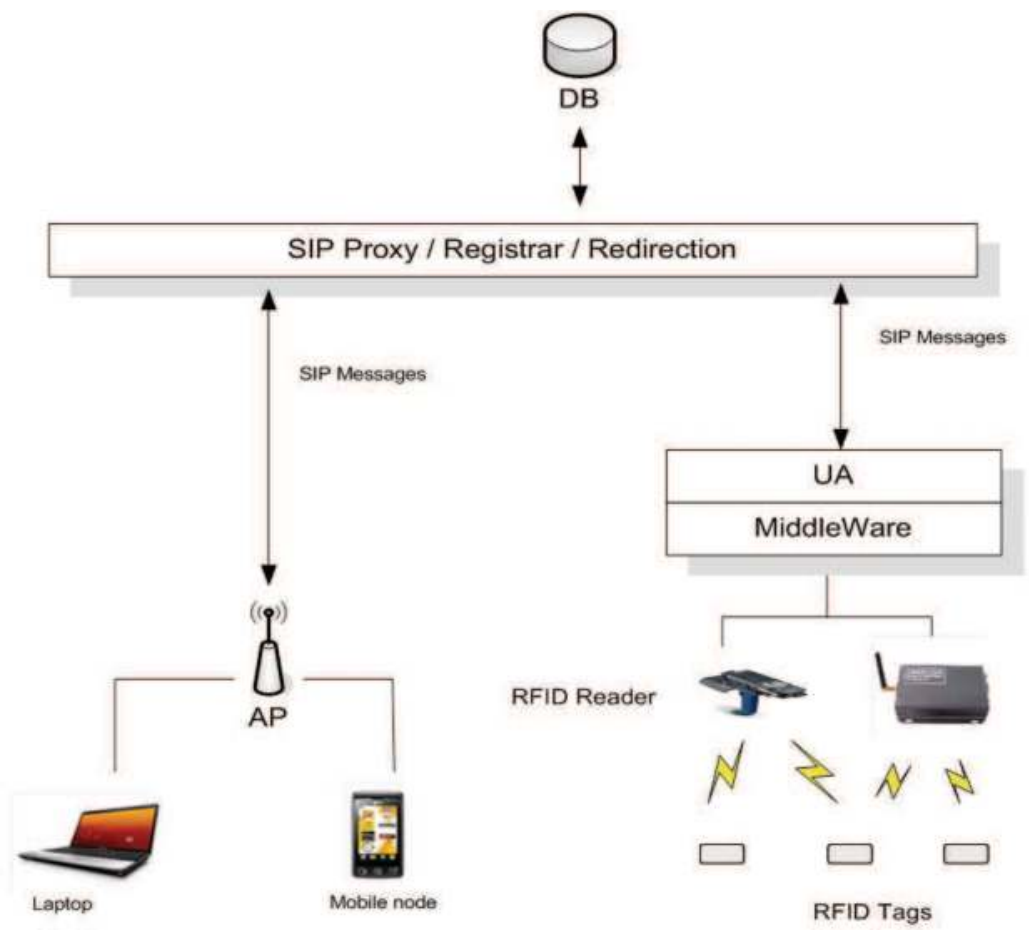

Fig. 2. SIP-based location system architecture 
As shown in the previous section, the location of a tag or object is mainly determined by the IP address of the reader to which it is attached.

In this specification, each RFID tag is associated with a SIP URI (Uniform Resource Identifier) which includes the RFID tag value and the reader's IP address to which this tag is attached. This URI reflects the current location of the RFID tag.

One of challenges of the RFID implementation is how to transform the RFID raw data into an understandable format which is directly used by the backend applications (e.g. tracking application). The location database is responsible for managing and generating the understandable format of location data.

Our location system database defines 4 main tables:

RFID-Location-Event table contains information on the RFID tag and its current network location. This information includes the RFID tag of the object, the IP address of the reader to which the tag is attached, and the timestamp which indicates the instant of detection of the tag object by the reader. An example of this table is given in table 1.

\begin{tabular}{|c|c|c|c|}
\hline Timestamp & RFID Tag & Object & IP Reader \\
\hline 0112200912012030 & 1a2e3f13ah & Enter & 137.194 .204 .69 \\
\hline 0112200913201734 & 1a2e3f43ed & Enter & 137.194 .204 .69 \\
\hline 0112200914012436 & 1a2e3f13ae & Leave & 137.194 .200 .80 \\
\hline 0112200918012445 & 1a2e4f5e6a & Enter & 137.194 .203 .100 \\
\hline
\end{tabular}

Table 1. RFID-Location-Event table example

RFID-Reader-Location table contains the geographical location of the RFID reader. This table maintains an association between the geographical location of the RFID reader and its IP address. An example of this table is given in table 2.

\begin{tabular}{|c|c|}
\hline IP Reader & Location \\
\hline 137.194 .204 .69 & Room C-208 \\
\hline 137.194 .200 .80 & Office 1B \\
\hline 137.194 .203 .100 & Lab. 103 \\
\hline
\end{tabular}

Table 2. RFID-Reader-Location table example

RFID-Name table contains the name of the object (or person) associated with the RFID tag (Table 3).

\begin{tabular}{|c|c|}
\hline RFID Tag & RFID Name \\
\hline 1a2e3f13ah & Dr. John \\
\hline 1a2e3f43ed & Mr. Smith (Patient) \\
\hline 1a2e3f13ae & Ms. Mary's Laptop \\
\hline 1a2e4f5e6a & Mrs. Rose's cell phone \\
\hline
\end{tabular}

Table 3. RFID-Name table example

RFID-DB table (an example given by Table 4) is generated by the Location Server when the tracking system has to determine the current location of the object. The Location Server combines the various tables depending on whether the tracking service uses the RFID tag or the object name as identity to look for their location. If, for example, the operation of the tracking searches the geographical location of a given object, the location engine combines the three tables: RFID-Location-Event, RFID-Reader-Location and RFID-Name to generate the current location of the RFID tags in the RFID-DB table. 


\begin{tabular}{|c|c|c|c|}
\hline RFID Name & RFID Tag & IP Reader & Location \\
\hline Dr. John & 1a2e3f13ah & 137.194 .204 .69 & Room C-208 \\
\hline
\end{tabular}

Table 4. RFID-DB table example

\subsection{Location function - SIP registration}

As mentioned above, the RCOM interface integrating the UA indicates the presence of tags in the detection zone of the reader by generating registration requests. SIP REGISTER messages are sent to the SIP server. Each SIP REGISTER message indicates in its Contact field the RFID tag and the IP address associated to the reader. The SIP server updates the location database. When an RFID tag leaves the area of the reader, a SIP REGISTER request with a field "Expires" $=0$ is generated.

Figure 3 represents the following registration procedure. As an illustration, we assume that an RFID tag is sticked to the business card of Dr. John who is a doctor at hospital A.

Step 1. When Dr. John arrives in the detection zone of a reader, the RFID tag information including the number of the RFID tag is collected.

Step 2. The reader transmits the obtained information to RFID middleware that performs filtering and collecting operations.

Step 3. The RCOM interface integrating the UA generates a SIP REGISTER message and sends it to the SIP server. The SIP REGISTER message contains the Contact field in an URI form which is the current location of the RFID tag (tag_RFID@IP_Reader). The server registers this information at the location database.

Step 4. If the registration is successful, the registrar generates a $200 \mathrm{OK}$ message to the UA. We assume, in this example, a registration without authentication. A SIP server configured only for authorized users generates a 407 SIP message type to request the credentials of the user before accepting the registration.

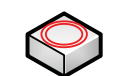

RFID Tag

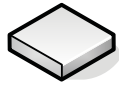

Reader

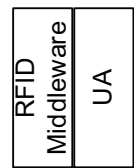

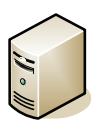

SIP Registrar

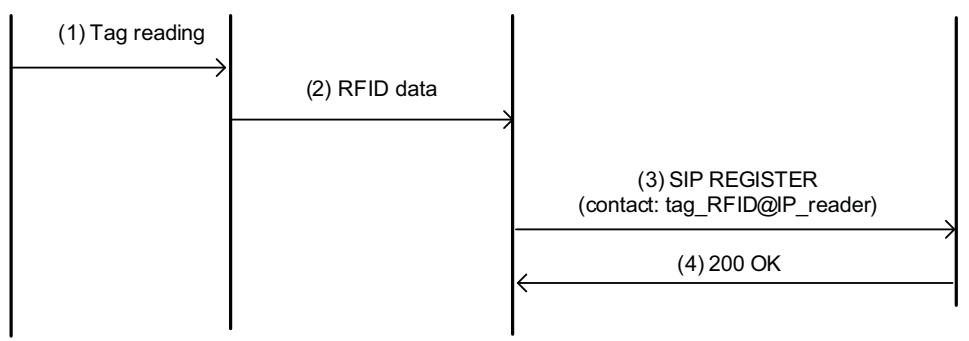

Fig. 3. Registration procedure

\subsection{Tracking function - SIP redirection}

To perform the tracking function, we assume that the UA is invoked by a location application which is out of scope of this specification. 
The UA generates a SIP INVITE message sent to the SIP server. The message contains a SIP URI to indicate the tag or object to locate. The SIP server acts as a redirect server that

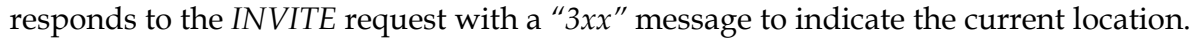

Figure 4 illustrates an example of the tracking procedure. In this example, a nurse wants to know where specific medical equipment is in the hospital.

The location application generates a SIP INVITE message. The SIP looks for the location of the object by consulting the location database and the redirect function notifies via a "302 Moved Temporarily" message, the current location of the equipment, for example, equipement_1@office_C208.

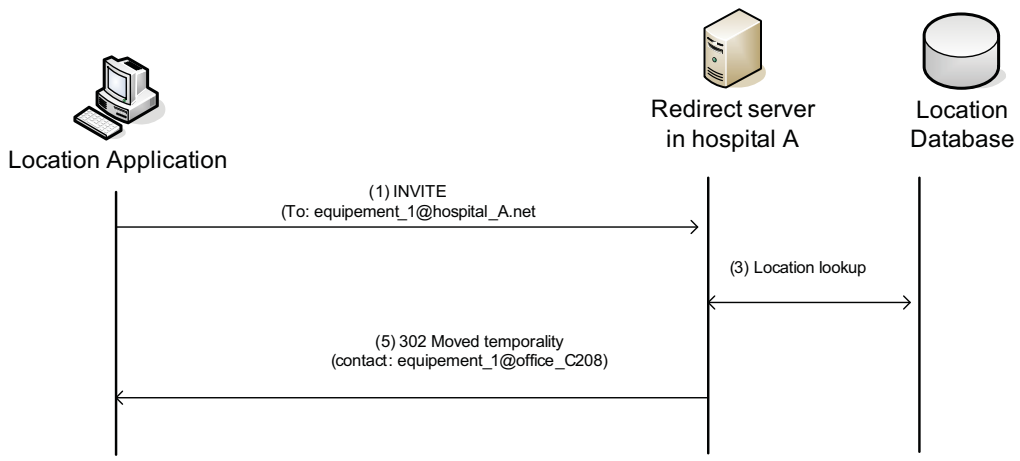

Fig. 4. Tracking procedure

\subsection{System implementation}

The location system is implemented under the Linux operating system and using the C programming language according to the open source GPL license. We implemented the system with some available tools of Linux environment such as PhidgetRFID card libraries and SIP express router (SER).

The PhidgetRFID card [phid] is an RFID reader which detects RFID tags that are brought in close of its proximity and returns the tag identification number. The PhidgetRFID reader uses the RFID EM4102 standard [em]. The PhidgetRFID libraries are APIs implemented in C under Linux that allow collecting the RFID tag and returning the unique number contained in the tag.

SIP Express Router (SER) [ser] is a high-performance, configurable, open-source GNU licensed server which can act as a SIP (RFC 3261 [sip]) registrar, proxy and redirect server. It provides many features of RFC 3261 functionality, a variety of database backends (mysql, oracle, etc.), management features (remote management via XML-RPC), NATi traversal, telephony features (LCR, speed dial), etc.

Figure 5 represents the system implementation that consists of the user agent (UA) with the location and tracking function, the SER server and the location database.

In our implementation, the backend location database uses My Structured Query Language (MySQL). We develop an UA based on the RFID source code of Phidget. We modified the SER source code so that to be able to receive/send the messages from UA.

The UA implementation have consisted in developing two events on the PhidgetRFID card for collecting the RFID tags and implementing a Location function to process the registration and tracking requests to the SIP server. 


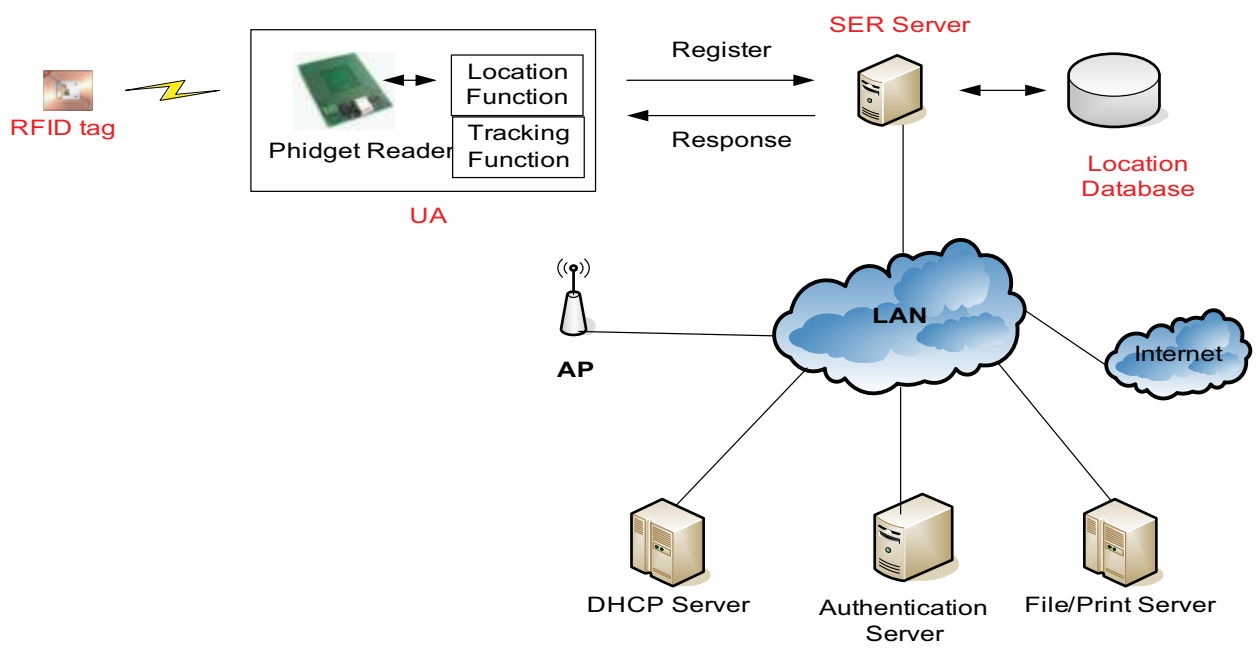

Fig. 5. The system implementation

In the following the main implemented functions are described:

int Register-Object(CPhidgetRFIDHandle RFID, void *usrptr, unsigned char *TagVal) // An event is issued by Phidget when a new tag is seen by the reader. The event is only fired one time for a new arrival of tag

int Deregister-Object(CPhidgetRFIDHandle RFID, void *usrptr, unsigned char *TagVal) // An event is issued by Phidget when a tag is out of the coverage zone of the reader.

char*Create_Request_Line(char *domain) // The request line specifies the type of request being issued by UA (e.g. SIP REGISTER or SIP INVITE), while the response line of SER server indicates the success or failure of a request (e.g. 200 OK, 302 Move temporality, ...).

char* Create_Contact(char *user_ip, char * user_port, char *user_name) // The contact field contains the current location of object. It indicates the corresponding <RFID tag, IP address of the RFID reader>

char*Create_Challenge_Register_Body (int seq, char *user_name, char *user_ip, char *user_port, char ${ }^{*}$ domain, char ${ }^{*}$ call_id, char ${ }^{*}$ tag) // This function generates the authentication message. The authentication uses the HTTP Digest method. The HTTP Digest authentication scheme is documented in [RFC3310] and extended in [RFC2617].

char*Create_Auth_Register(char *nonce, int seq, char *user_name, char *user_ip, char *user_port, char *domain, char *call_id, char *tag, char *user_password); // This function generates the authentication response message.

\section{Conclusion}

In this chapter, we proposed an IP-based RFID architecture that allows low cost and large scale deployment, as well as easy integration with IP-based services.

Particularly, a location management support is provided. The RCOM interface is introduced to handle location registration messages and entering/leaving tags detection. 
A SIP-based architecture and the system implementation have been proposed for validation purpose.

This work is a part of an overall project which aims at developing a global communication network including RFIDs and IP entities inthe effort to contribute the realization of the future Internet of things.

\section{References}

[aero] Aeroscout solutions - [online] http://www.aeroscout.com

[cho] Cho, K. Pack, S. Kwon, T. Choi, Y. (2007), SRMS: SIP-based RFID Management System, Proceedings of IEEE International Conference on Pervasive Services, pp. 11-18, ISBN: 1-4244-1325-7, Istanbul (Turkey), 15-20 Jul. 2007.

[edoc] Ekahau Whitepaper (2005) - Comparison of Wireless Indoor Positioning Technologies - $\quad$ [online]http://www.productivet.com/docs2/Wireless_Comparison.pdf

[ekah] Ekahau Solutions - [online]http://www.ekahau.com

[em] EM4102 protocol - [online]http://www.ibtechnology.co.uk/PDF/EM4102_DS.pdf

[epc] EPCglobal Website, [online]http://www.epcglobalinc.org.

[high] J. Hightower, C. Vakili, C. Borriello and R. Want (2001), Design and calibration of the SpotON ad-hoc location sensing system, UW CSE 00-02-02, University of Washington, Department of Computer Science and Engineering, Seattle, WA, August 2001.

[itu] ITU Internet Report (2005) - The Internet of Things, - [online] http://www.itu.int/dms_pub/itu-s/opb/pol/S-POL-IR.IT-2005-SUM-PDF-E.pdf

[ivan] Getting, Ivan A (1993), The Global Positioning System, In: IEEE Spectrum, pp. 36 - 37, December 1993.

[joy] Joy, V. Vimal Laxman, P. (2007), Smart Spaces: Indoor Wireless Location Management System, Proceedings of Next Generation Mobile Applications, Services and Technologies (NGMAST), pp. 261-266, ISBN: 978-0-7695-2878-6, Cardiff, 12-14 Sept. 2007.

[jud] Judith M. Myerson (2006), RFID in the Supply Chain: A Guide to Selection and Implementation, ISBN / ASIN: 0849330181, AUERBACH, 20 Nov. 2006

[klau] Klaus F. (2003), RFID Handbook: Fundamentals and Applications in Contactless Smart Cards and Identification, 2nd Edition, ISBN / ASIN: 0470844027, Wiley, 23 May 2003

[liu] X. Liu, M.D. Corner, and P. Shenoy (2006), Ferret: RFID Localization for Pervasive Multimedia, In P. Dourish, and A. Friday (Eds.), International Conference of Ubiquitous Computing (Ubicomp 2006). LNCS 4206, pp. 422-440, 2006.

[nad] Boukhatem, N. Tran, P.N. (2008), IP-based RFID architecture and location management, Proceedings of the 16th International Conference on Software, Telecommunications and Computer Networks (SoftCOM), pp. 95-99, ISBN: 978-9536114-97 9, Split (Croatia), 25-27 Sept. 2008.

[ni] L. Ni , Y. Liu , Y. Cho Lau, A. Patil (2004), LANDMARC: Indoor Location Sensing Using Active RFID. In Wireless Networks 10, pp.701-710, Kluwer Academic Publishers. Netherlands, 2004.

[ohta] Y. Ohta, M. Sugano, and M. Murata (2005), Autonomous Localization Method in 
Wireless Sensor Networks, Proceedings of 3rd IEEE International Conference on Pervasive Computing and Communications Workshops 2005 (PerCom 2005), Kauai Island, USA, 2005.

[phid] PhidgetRFID card - [online] http://www.phidgets.com/products.php?category=14\&product_id=1023

[RFC3310] Hypertext Transfer Protocol (HTTP) Digest Authentication Using Authentication and Key Agreement (AKA) - [online] ftp://ftp.isi.edu/innotes/rfc3310.txt

[RFC2617] HTTP Authentication: Basic and Digest Access Authentication - [online] ftp://ftp.isi.edu/in-notes/rfc2617.txt

[ser] SIP Express Router - [online] http://www.iptel.org/ser

[sip] SIP: Session Initiation Protocol - RFC3261- [online] http://www.ietf.org/rfc/rfc3261.txt

[sue] K.L. Sue, C.H. Tsai, and M.H. Lin (2006), FLEXOR: A Flexible Localization Scheme Based on RFID, In I. Chong, and K. Kawahara (Eds.), International Conference on Information Networking (ICOIN), LNCS 3961, pp. 306-316, 2006.

[teso] R. Tesoriero, R. Tebar, J.A. Gallud, M.D. Lozano, V.M.R. Penichet (2009), Improving location awareness in indoor spaces using RFID technology, In Expert Systems with Applications Journal, Vol. 37 (2010), pp. 894-898

[wein] Weinstein, R. (2005), RFID: a technical overview and its application to the enterprise, In: IT Professional, Volume: 7, Issue: 3, pp. 27- 33, ISSN: 1520-9202, May-June 2005.

[yang] Q. Yang, Y. Chen, J. Yn, and X. Chai (2004), LEAPS: A Location Estimation and Action Prediction System in a Wireless LAN Environment, In H. Jin et al. (Eds.), IFIP International Conference on Network and Parallel Computing (NPC 2004), LNCS 3222, pp. 584-591, 2004.

[zang] Zang, L. Chao-Hsien, C. Wen, Y. (2008), SIP-RLTS: An RFID Location Tracking System Based on SIP, Proceedings of IEEE International Conference on RFID, pp. 173182, Las Vegas (USA), 978-1-4244-1711-7, 16-17 Apr. 12008.

[zhang] Zhang T., Xiong Z., Ouyang Y. (2006), A Framework of Networked RFID System Supporting Location Tracking, Proceedings of the 2nd IEEE/IFIP International Conference in Central Asia on Internet, ISBN: 1-4244-0543-2, pp. 1-4, Tashkent, Uzbekistan, 19 -21 September 2006.

[weiser] M. Weiser (1991), The computer of the 21st century, Scientific American, pages 94100, September 1991. 


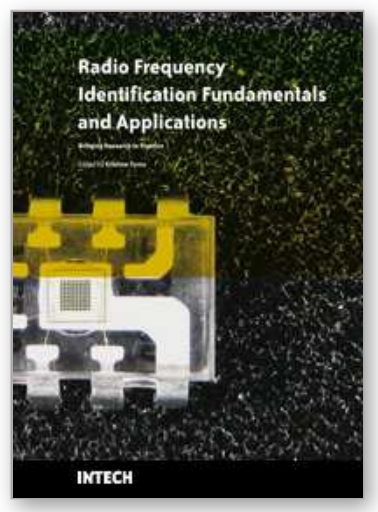

\author{
Radio Frequency Identification Fundamentals and Applications \\ Bringing Research to Practice \\ Edited by Cristina Turcu
}

ISBN 978-953-7619-73-2

Hard cover, 278 pages

Publisher InTech

Published online 01, February, 2010

Published in print edition February, 2010

The number of different applications for RFID systems is increasing each year and various research directions have been developed to improve the performance of these systems. With this book InTech continues a series of publications dedicated to the latest research results in the RFID field, supporting the further development of RFID. One of the best ways of documenting within the domain of RFID technology is to analyze and learn from those who have trodden the RFID path. This book is a very rich collection of articles written by researchers, teachers, engineers, and professionals with a strong background in the RFID area.

\title{
How to reference
}

In order to correctly reference this scholarly work, feel free to copy and paste the following:

Phuoc Nguyen Tran and Nadia Boukhatem (2010). IP-based RFID Location System, Radio Frequency Identification Fundamentals and Applications Bringing Research to Practice, Cristina Turcu (Ed.), ISBN: 978953-7619-73-2, InTech, Available from: http://www.intechopen.com/books/radio-frequency-identificationfundamentals-and-applications-bringing-research-to-practice/ip-based-rfid-location-system

\section{INTECH}

open science | open minds

\section{InTech Europe}

University Campus STeP Ri

Slavka Krautzeka 83/A

51000 Rijeka, Croatia

Phone: +385 (51) 770447

Fax: +385 (51) 686166

www.intechopen.com

\section{InTech China}

Unit 405, Office Block, Hotel Equatorial Shanghai

No.65, Yan An Road (West), Shanghai, 200040, China

中国上海市延安西路 65 号上海国际贵都大饭店办公楼 405 单元

Phone: +86-21-62489820

Fax: $+86-21-62489821$ 
(C) 2010 The Author(s). Licensee IntechOpen. This chapter is distributed under the terms of the Creative Commons Attribution-NonCommercialShareAlike-3.0 License, which permits use, distribution and reproduction for non-commercial purposes, provided the original is properly cited and derivative works building on this content are distributed under the same license. 